\title{
Role of PAR1 in Food Allergies
}

\author{
Jennifer Garcia1 ${ }^{1}$ Lauren Buelow², Joan Cook-Mills², \\ Indiana University School of Medicine ${ }^{1}$; Indiana University School of Medicine, Department of \\ Microbiology and Immunology ${ }^{2}$
}

Background and Hypothesis:

The prevalence of food allergies continues to rise. In a mouse model, food allergy to peanuts develops in flaky tail mice with skin barrier mutations and exposure to peanut (PNE) and Alternaria Alternata (fungal allergen, Alt) on the skin. In the skin, keratinocytes respond to proteases in allergens through protease activating receptor 1 (PAR1). Blocking PAR1 decreased the severity of viral induced inflammation in mice. Whether PAR1 has a major role in food allergies has not been investigated. We tested the hypothesis that blocking PAR1 would halt the development of food allergy to peanuts in neonatal mice.

Project Methods:

In our studies, pups were injected intradermally (i.d.) with a PAR1 antagonist and then treated with PNE/Alt. In another group, pups received i.d. injections of a PAR1 agonist and then treated with PNE only. Control groups received allergens only. Pups were treated and skin sensitized 5 times every 3-4 days. Forty-eight hours after the last treatment, pups were challenged with PNE through oral gavage, and temperatures were recorded every 15-30 minutes for 3 hours. Skin, ileum, and jejunum samples were collected and used for qPCR to determine the expression of inflammatory mediators. Plasma serum was used for analysis of anti-PNE specific antibodies by ELISA.

Results:

PAR1 antagonist blocked anaphylaxis in allergic mice sensitized with PNE and Alt. PAR1 agonist is sufficient to induce anaphylaxis in mice sensitized with PNE only.

Conclusion and Potential Impact:

This study demonstrates that PAR1 is involved in the development of food allergies, where blocking the receptor blocked food allergies in neonatal mice. The signaling mechanisms and activators of PAR1 need further studies, using PAR1 deficient mice. This novel pathway may lead to therapies to stop the development of food allergies. 